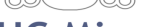

PUC Minas

E\&G - REVISTA ECONOMIA E GESTÃO

ISSN 1984-6606

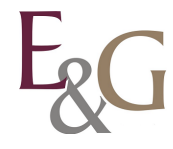

\title{
Gestão estratégica do conhecimento: um estudo comparativo em empresas incubadas
}

\section{Strategic knowledge management: a comparative study in incubators of business}

\author{
Prof ${ }^{a}$. Ms. Claudia Andressa Cruz \\ andressaufscar@yahoo.com.br \\ Professora Mestre do Centro Universitário Central Paulista (UNICEP) e da Faculdade Anhanguera de Matão \\ Prof. Dr. Marcelo Seido Nagano \\ drnagano@usp.br \\ Professor Doutor do Departamento de Engenharia de Produção - Escola de Engenharia de São Carlos - \\ Universidade de São Paulo \\ Editor Científico Responsável: Prof. Dr. Cândido Borges \\ Professor do curso de Administração da Universidade Federal de Goiás
}

Recebido em: 30 de abril de 2009

Aprovado em: 24 de março de 2010

\begin{abstract}
Resumo
O presente trabalho tem o objetivo de explorar o comportamento das variáveis inerentes à gestão estratégica do conhecimento, em empresas incubadas no Estado de São Paulo. Com intuito de atingir o objetivo proposto, buscou-se identificar as principais fases do processo de gestão do conhecimento (socialização, externalização, combinação e internalização) e qualificá-las por meio de atributos; e identificar as dimensões estratégicas (cultura, práticas de recursos humanos e sistemas de informação) que estejam relacionadas à gestão do conhecimento. Evidenciadas essas variáveis teóricas, foi realizada a pesquisa empírica mediante um questionário fechado, possibilitando quantificar os resultados, destinado aos dirigentes dessas empresas. A análise permitiu explorar as principais diferenças entre as duas incubadoras, destacando as seguintes práticas: treinamento, interações coletivas e virtuais, utilização de banco de dados e elaboração de relatórios. Evidenciou-se um conhecimento mais sistematizado em um primeiro grupo de empresas, em contrapartida ao outro grupo, que apresentou uma cultura de incentivo ao acesso dos bancos mais intenso e interações mais individualizadas.
\end{abstract}

Palavras-chave: Gestão do conhecimento. Estratégia. Incubadoras de empresas. Gestão estratégica. Gestão estratégica do conhecimento.

\footnotetext{
Abstract

This paper aims to explore the behaviour of the strategic knowledge management incubated at São Paulo's State. In order to achieve this purpose, aims to: identify the main stages of knowledge management (socialization, outsourcing, combination and internalization), and to classify them by attributes, however, identify the strategic dimensions characterized and identify strategic dimensions (culture, human resources practices and information systems) that are related to knowledge management. Evidenced these theoretical variables, it was conducted an empirical research through a closed questionnaire, allowing quantify the results, for the company's leaders. The analysis allowed exploring the main differences between the two incubators, highlighting, and the
} 
following practices: training, collective and virtual interactions, use of database and reporting. Exposing a more systematized knowledge into a first group of companies, however the other group with a culture to encourage the banks access to more intense and individualized interactions.

Keywords: Knowledge management. Strategy. Incubators. Strategic management. Strategic knowledge management.

\section{INTRODUÇÃO}

Notadamente, a administração é consagrada por diversos estudos gerenciais que, embora incorporem aspectos organizacionais distintos, possuem a mesma finalidade, proporcionar lucratividade às organizações industriais. De acordo com Wren (2007), o pensamento administrativo pode ser caracterizado por três grandes períodos, em que cada um é caracterizado de acordo com o predomínio das ideias naquele momento. O primeiro período, composto por Administração Científica e Teoria Clássica, destaca a busca por eficiência e racionalização do trabalho (PARKER; RITSON, 2005); a Era Social ou Relações humanas marca o segundo período com a introdução de noções de liderança e os grupos informais; e o terceiro grande momento denota a necessidade de as organizações se adaptarem constantemente ao ambiente em que estão inseridas.

Um novo período é caracterizado à medida que se destaca a necessidade de mecanismos, tais como a inovação, o desenvolvimento de competências, novas formas de gestão e a reestruturação organizacional, para lidar com o aumento na taxa de criação e mortalidade de empresas (TERRA, 2001). Dessa forma, ocorre uma transição em que o poder de gerar a riqueza e o poder da sociedade atual deixam de provir, exclusivamente, dos fatores tradicionais de produção, tais como capital, terra e trabalho, advindos também de bens intangíveis, como o conhecimento organizacional (LEONARD-BARTON, 1998; SANTIAGO JR., 2002).

Nonaka (1991) menciona que a única certeza desse período é a incerteza e que a única fonte de vantagem competitiva duradoura é o conhecimento, portanto o gerenciamento estratégico do conhecimento seria uma alternativa na busca por vantagens competitivas.

A gestão estratégica do conhecimento (GEC) é composta, neste trabalho, pelo processo de gestão do conhecimento (GC), somado às dimensões estratégicas organizacionais, ou melhor, funções organizacionais que viabilizem a gestão do conhecimento nas empresas. Segundo Nonaka, Peltokorpi e Tomae (2005), a gestão estratégica pode ser vista como um

processo mecanicista ou orgânico que corresponde, respectivamente, à escola do 
posicionamento que produz serviços e produtos baseados na análise de mercado, portanto do ambiente externo (PORTER, 2003); e à visão baseada em recursos (VBR) que prioriza o acúmulo de recursos internos (WERNERFELT, 1984). Dessa forma, gestão estratégica do conhecimento analisa que tanto os fatores exógenos (externos) quanto os endógenos (internos) devem ser considerados.

O avanço científico-tecnológico impôs novos padrões de desenvolvimento econômico. A flexibilização e a automação do processo produtivo provocaram alterações significativas nas denominadas economias de escala. A necessidade de mudanças organizacionais, em virtude da concorrência acirrada ou diferentes estágios do ambiente empresarial, remete à reflexão de que uma gestão estratégica do conhecimento pode ser uma alternativa na busca por vantagem competitiva (FLEURY; OLIVEIRA JR., 2001).

Uma consequência desses novos padrões de desenvolvimento é a criação de incubadoras de empresas, ambientes que visam promover o desenvolvimento econômico e a inovação a partir da retenção de empregos com alto valor agregado, comercialização de novas tecnologias e promoção de relações entre a indústria, o governo e a academia (MEDEIROS et al., 1992). Com intuito de analisar esse ambiente, a análise da gestão estratégica do conhecimento, neste trabalho, é realizada em empresas incubadas, por estas fomentarem o desenvolvimento tecnológico, apoiado na gestão eficaz do conhecimento individual e organizacional.

Diante desse contexto, o principal objetivo desta pesquisa é explorar comparativamente o comportamento das variáveis inerentes à gestão estratégica do conhecimento em duas incubadoras paulistas.

Fundamentado no exposto e diante da importância de se conhecerem os aspectos e as dinâmicas nesses grupos diferenciados de empresas, foi realizada uma pesquisa de natureza exploratória e quantitativa, com base em elementos identificados na literatura como sendo essenciais à gestão do conhecimento e à administração estratégica, com o objetivo de demonstrar as similaridades e as disparidades entre os dois núcleos investigados.

\section{GESTÃO ESTRATÉGICA DO CONHECIMENTO}

A gestão do conhecimento, especificamente, visa conquistar acertos organizacionais por meio de estratégia dirigida para a motivação e a facilitação do conhecimento dos trabalhadores. Essa estratégia visa criar, disseminar, armazenar e incorporar às rotinas de 
PUC Minas

trabalho todo o conhecimento desenvolvido na organização, utilizando a capacidade dos trabalhadores para interpretação de dados e informações (por recursos disponíveis de informação, experiências, habilidades, cultura, características, personalidade, sentimentos entre outros) (BEIJERSE, 1999).

A gestão do conhecimento é analisada por Nonaka (1991) como um ciclo de criação contínuo do conhecimento, visto pelo autor como a criação do conhecimento organizacional, observado nas companhias bem sucedidas que criam novos conhecimentos, disseminam-nos amplamente por toda organização e rapidamente os incorporam em novos produtos, serviços e tecnologias. A teoria de criação do conhecimento é caracterizada como um processo que envolve interações entre os vários níveis organizacionais, a partir da transformação do conhecimento tácito (pessoal e informal) em conhecimento explícito (formal e sistemático), processo denominado SECI - Socialização, Externalização, Combinação e Internalização.

O processo de gestão de conhecimento, SECI, compõe assim os fatores exógenos e endógenos das empresas (QUADRO 1), por considerar não apenas o processo interno de gestão do conhecimento, mediante o desenvolvimento de práticas que fomentam o ciclo contínuo de ampliação do conhecimento mas também os processos inerentes à organização, como os mecanismos de transferência externa, a partir de fornecedores, clientes e parceiros.

\begin{tabular}{|c|c|}
\hline Socialização & Externalização \\
\hline $\begin{array}{l}\text { Interaçăo individual face a face } \\
\text { Treinamento } \\
\text { Confiança } \\
\text { Comprometimento }\end{array}$ & $\begin{array}{l}\text { Interaçóes coletivas face a face } \\
\text { Conceito de produto /Analogia } \\
\text { Incentivo a criatividade e diálogo }\end{array}$ \\
\hline Internalização & Combinação \\
\hline $\begin{array}{l}\text { Interaçóes individuais e virtuais } \\
\text { Cultura organizacional disseminada } \\
\text { Know how técnico } \\
\text { Learning by doing }\end{array}$ & $\begin{array}{l}\text { Interaçóes coletivas e virtuais } \\
\text { Banco de dados } \\
\text { Redes de comunicaçảo } \\
\text { Elaboraçăo de relatórios }\end{array}$ \\
\hline
\end{tabular}

Quadro 1: Fases e atributos do processo SECI

Fonte: CRUZ, 2007.

Do exposto é possível inferir que a fase de socialização se caracteriza por interação face-a-face (NONAKA, 1994; SILVA, 2004), indispensável nessa conversão; e por uma cultura de confiança e comprometimento (CARDOSO, 2003; NONAKA; TOYAMA; KONNO, 2000), uma vez que atuam como facilitadores, ao evitarem que haja qualquer receio em compartilhar conhecimento com o próximo; ademais, a ação que facilita efetivamente o 
compartilhamento de conhecimentos tácitos é o treinamento (NONAKA; KONNO, 1998; TEECE, 1998).

A externalização abrange basicamente as interações coletivas e face-a-face (NONAKA; TAKEUCHI, 1997), facilitando as atividades em equipe e permitindo um leque maior de conhecimentos distintos a serem compartilhados; e a utilização de analogias e metáforas que transmitam novos conceitos de produtos, o que é amplamente verificado em atividades de desenvolvimento de produtos (NONAKA, 1994; SILVA, 2002), em que a criatividade e o diálogo devem ser incentivados, uma vez que a abstração dos conceitos pode direcionar a inovações importantes de bens e serviços.

$\mathrm{Na}$ combinação, inicia-se a incorporação de alguns aspectos das tecnologias de informação, e esse processo remete às interações coletivas e virtuais (NONAKA; KONNO, 1998; NONAKA; TOYAMA; NAGATA, 2000), em que ambientes cibernéticos servem como meio para a troca de conhecimento de vários indivíduos.

Não obstante esses aspectos, a elaboração de relatórios que armazenem em banco de dados todo o conhecimento aprendido deve ser priorizado nessa etapa; desse modo, as redes de comunicação auxiliam no armazenamento, disponibilização e transferência de conhecimento (COHENDET; STEINMUELLER, 2000; COWAN; DAVID; FORAY, 2000; NONAKA; TAKEUCHI, 1997).

E, por fim, os atributos mais relacionados à internalização são as interações individuais e virtuais (NONAKA; KONNO, 1998), uma vez que a consulta de registros de conhecimento são indispensáveis para o aprendizado pessoal (SILVA, 2004); o know-how técnico e o learning by doing, facilitadores dessa etapa; e, como ação gerencial, uma cultura organizacional disseminada, que serve como motivadora da incorporação de todo conhecimento adquirido (NONAKA; TAKEUCHI, 1997).

Somando-se a isso, as dimensões estratégicas configuram os fatores endógenos que subsidiam esse processo, e as que se destacam neste trabalho são: uma cultura organizacional que permeie a confiança para o compartilhamento do conhecimento individual; sistemas de informação, como provedores de suporte técnico e funcionalidade que oferecem ao processo de gestão do conhecimento; e práticas de recursos humanos, vislumbrando um alto comprometimento da empresa com os membros organizacionais, visando atrair, reter, motivar e encorajar os membros da organização.

Um dos grandes entraves ao compartilhamento do conhecimento é a tendência das pessoas em quererem guardar seus conhecimentos, dificultando sobremaneira o ato de compartilhamento, o que, sem o apropriado gerenciamento estratégico, dificilmente fará do 
conhecimento um diferencial competitivo. Nesse sentido, a cultura deve voltar-se para a conscientização dos empregados, o incentivo e o reconhecimento (BENÍTEZ; RODRIGUEZ; RODRIGUEZ, 2004).

A cultura organizacional tem como função viabilizar a clareza das missões e dos valores organizacionais por meio de atos simbólicos; e o compartilhamento de conhecimento e a experimentação a partir do comprometimento e reconhecimento dos funcionários, estabelecendo uma cultura de confiança e liberdade, satisfazendo assim as necessidades e as aspirações dos membros da organização, e incentivando a exploração de territórios desconhecidos, tais como novos mercados, novos clientes, produtos e novas tecnologias de manufatura (DAVENPORT; DE LONG; BEERS, 1998; NONAKA, 1994; NONAKA; UMEMOTO; SENOO, 1996; ORSI, 2003; TERRA, 2001; TIPARK, 2005; BEIJERSE, 1999; KROGH, 1998; ZACK, 1999).

Devido ao suporte técnico e a toda funcionalidade que os sistemas de informação oferecem ao processo de gestão do conhecimento, consideram-se também aqui os sistemas de informação como uma das dimensões estratégicas. Os recursos da tecnologia de informação podem otimizar fases do processo SECI, seja por facilitar que as pessoas sejam encontradas, podendo ocorrer então a socialização e externalização; seja por auxiliar no registro do conhecimento, na combinação; ou agilizar o acesso ao conhecimento, na internalização (SCHULZ, 2001; SILVA, 2002).

Assim, investimentos em tecnologias de informação e integração dos sistemas tornamse indispensáveis na facilitação de transferência de informações, e, principalmente, de conhecimento, sem contar o modo como facilitam o processo de codificação do conhecimento, configurando um importante aspecto estratégico (COWAN; DAVID; FORAY, 2000; COHENDET; STEINMUELLER, 2000; DAVENPORT; DE LONG; BEERS, 1998; HANSEN; NOHRIA; TIERNEY, 1999; HITT; IRELAND; HOSKINSSON, 2002; PRAHALAD; HAMEL, 1990; TERRA, 2001; VASCONCELOS, 2001; ZACK, 1999).

A última dimensão estratégica a ser considerada nesta pesquisa são as práticas de recursos humanos que envolvem, principalmente, reconhecimento, recompensa por meio de políticas de compensação, treinamento e admissão de novas competências.

Storey e Quintas (2001) e Campos, Ferreira e Silva (2009) corroboram ao destacar que para obter-se uma gestão do conhecimento efetiva é necessário um alto comprometimento da empresa com estratégias de inovação; para tanto, as estruturas organizacionais devem ser redesenhadas, assim como uma nova cultura organizacional deve se estabelecer. Isso perante as práticas de recursos humanos implica novas prioridades em recrutamento/seleção/equipes; 
PUC Minas

nova ênfase no aprendizado organizacional; e revisão completa da gestão de desempenho e sistemas de recompensas.

Medeiros (2003) corrobora com os atributos supracitados inseridos nas políticas de recursos humanos ao analisar, em seu estudo, contratação, carreira, remuneração, treinamento e avaliação de desempenho, as quais estão diretamente relacionadas com as que serão estudadas, com exceção das variáveis carreira e avaliação de desempenho. Esta última, embora não seja pesquisada diretamente, está relacionada indiretamente com as políticas de compensação que são proporcionais ao desempenho.

Nesse sentido, esta pesquisa analisa as relações dos fatores endógenos (cultura, políticas de recursos humanos e os sistemas de informação) com o processo de gestão de conhecimento, que considera não apenas os processos inerentes à organização, como os mecanismos de transferência externa, a partir de fornecedores, clientes e parceiros (FIG. 1).

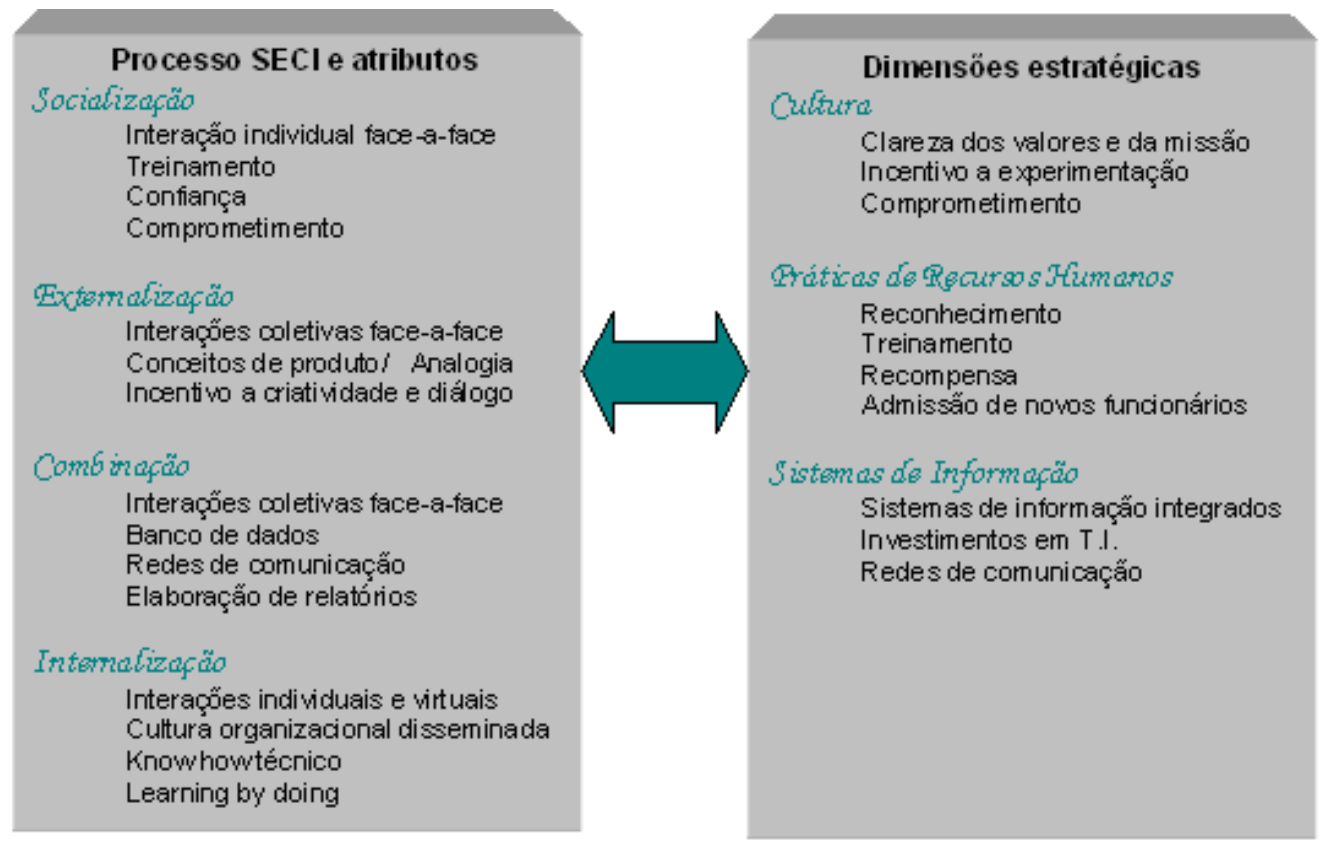

Figura 1: Relacionamento entre os fatores do processo SECI e as dimensões estratégicas Fonte: CRUZ, 2007.

Dessa forma, a Figura 1 mostra os fatores intrínsecos à gestão estratégica do conhecimento, denotando que a relação entre o processo de criação do conhecimento SECI e as dimensões organizacionais que subsidiam esse processo constituem uma relação dinâmica e concomitante representada pela seta dupla. 
PUC Minas E\&G - REVISTA ECONOMIA E GESTÃO

ISSN 1984-6606

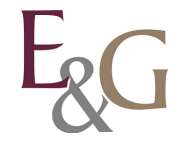

A composição dos fatores apresentados na Figura 1, variáveis base de estudo neste artigo, foi consolidada após extensa revisão bibliográfica e pesquisa empírica, conforme Cruz (2007).

Este trabalho visa explorar a dinâmica desses fatores apontados na Figura 1 nas pequenas empresas e não no contexto das grandes organizações, como fazem muitas teorias. As empresas de pequeno porte, no Brasil e no mundo, representam um papel importante na economia, uma vez que apresentam grande participação na distribuição de renda, na absorção de grande parte da mão-de-obra, na geração de novos postos de trabalho (PREVIDELLI; MEURER, 2005).

No contexto socioeconômico brasileiro, micro e pequenas empresas são extremamente relevantes, pois são identificadas como fator de promoção social, devido a sua capacidade de gerar empregos e incorporar tecnologia (TERENCE, 2002).

Segundo o IBGE (2005), no Brasil existem 4,6 milhões de empresas, desse total, 99\% são micros e pequenas empresas. Os pequenos negócios (formais e informais) respondem por mais de dois terços das ocupações do setor privado, conforme mostra o Gráfico 1, entretanto as MPE são responsáveis por apenas $20 \%$ do PIB.

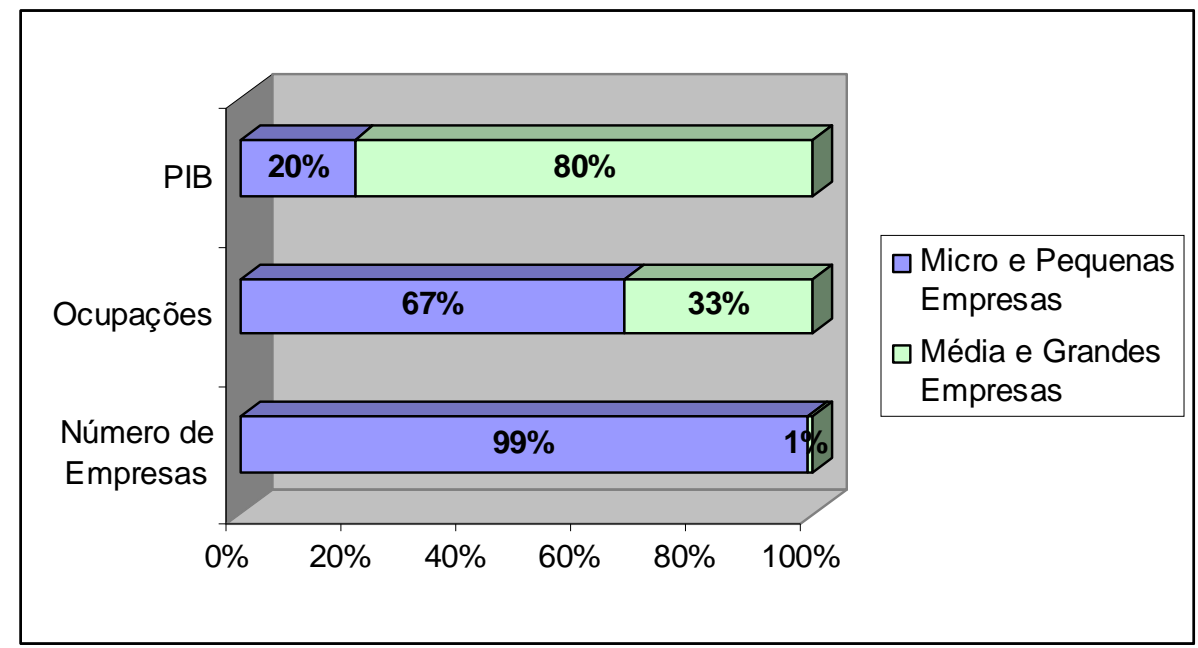

Gráfico 1: A presença das micro e pequenas empresas na economia brasileira Fonte: IBGE, 2005. Adaptado.

De acordo com Leone (1999), as pequenas empresas possuem problemas distintos em comparação às grandes empresas, merecendo, desse modo, uma teoria específica a seu contexto. Lima e Zoschke (2007) corroboram quando mencionam também a necessidade de entendimento das relações internas nas pequenas empresas. 
Possivelmente, algumas dessas especificidades das pequenas empresas devem ser compatíveis com aquelas encontradas nas incubadoras de empresas, tendo em vista o ambiente de alta tecnologia e de integração com o conhecimento acadêmico em que estas estão inseridas.

Segundo Medeiros et al. (1992), incubadoras de empresas são constituídas por empresas reunidas num mesmo local, dentro do campus da universidade, ao lado deste ou em uma área próxima. Conceitua que, instaladas nesses ambientes, encontram-se empresas de base tecnológica caracterizadas por incorporar o conhecimento científico-tecnológico como seu principal insumo de produção e por relacionarem-se intensivamente entre si e com a universidade ou instituto de pesquisa.

As incubadoras de empresas têm o objetivo de criar, em um ambiente propício, a inovação, via integração num único ambiente de universidades, empresas tradicionais e de base tecnológica e agentes de fomento (TORKOMIAN, 1992).

Nesse contexto, é possível observar que as empresas instaladas em incubadoras de empresas, objeto de estudo deste trabalho, são consideradas ambientes adequados para investigação do processo de gestão do conhecimento, por contribuírem com o desenvolvimento e capacitação regional por meio do conhecimento científico-tecnológico.

Outro aspecto relevante desta pesquisa está na busca de elementos que viabilizem o entendimento das relações entre os fatores do processo de gestão do conhecimento e dimensões estratégicas organizacionais.

Em geral, na literatura especializada, os fatores do processo de gestão do conhecimento são analisados independentemente das dimensões estratégicas que os subsidiam, por isso a importância desta pesquisa em entender, de forma agregada, os mecanismos que preenchem a lacuna entre esses elementos.

A relevância desta pesquisa é dada também pelo fato de poder contribuir com a Lei de Inovação Tecnológica - Lei Federal n. 10.973/2004 (BRASIL, 2005), que tem como objetivo criar um ambiente propício ao aumento do envolvimento das empresas no desenvolvimento de projetos inovadores que levem à geração de novos produtos e processos. Essa lei busca uma elevação do nível de parcerias entre empresas, universidades e institutos científicos e tecnológicos para que ganhem força e estimulem o processo de inovação (MATIASPEREIRA; KRUGLIANKAS, 2005).

\section{MÉTODO DE PESQUISA}


A análise do relacionamento entre os fatores relativos à gestão do conhecimento e às dimensões estratégicas em empresas incubadas é um tema pouco explorado na literatura, o que caracteriza uma pesquisa exploratória e descritiva, pois realiza descrições categóricas da situação, visando descobrir as relações existentes entre os elementos componentes desta, e explora um assunto pouco abordado, medindo as características descritas nas questões de pesquisa (CERVO; BERVIAN, 1983; DANE, 1990; GIL, 2002; HAIR JR et al., 2005a; LAKATOS; MARCONI, 1992).

Foi realizada uma análise nas empresas instaladas em duas incubadoras pertencentes ao Estado de São Paulo: o Parque Científico e Tecnológico de Campinas - Ciatec (Cia. de Desenvolvimento do Pólo de Alta Tecnologia de Campinas), localizado na cidade de Campinas; e o Parque Tecnológico de São Carlos - ParqTec, localizado na cidade de São Carlos. A população-alvo é composta por 39 empresas incubadas. A composição das empresas por incubadora configura-se da seguinte forma: 23 empresas na Cia. de Desenvolvimento do Polo de Alta Tecnologia de Campinas (Ciatec); e 16 empresas no Parque Tecnológico de São Carlos ( ParqTec).

A escolha de uma população constituída por empresas incubadas remete à magnitude de conhecimento incorporado nestas; trata-se de empresas que estão constantemente inovando, formadas, em sua maioria, por pessoas altamente especializadas em sua área de atuação, e, ademais, há transferência contínua de conhecimento das universidades-empresas devido ao contexto que estão inseridas.

Não obstante esses aspectos, sabe-se que diferenças estruturais, pessoal e gestão, entre outras, são comuns entre as empresas. A abordagem quantitativa desta pesquisa permite visualizar a existência ou não de diferenças significativas em termos de gestão do conhecimento entre os grupos de empresas pesquisadas: ParqTec e Ciatec.

Para a coleta de dados, a técnica adotada foi o envio de um questionário fechado, por meio de correio eletrônico, aos dirigentes das empresas. O questionário empregou a escala Likert, contida no intervalo de 1 a 7 , em que 1 corresponde à alternativa "discordo totalmente" e 7 à "concordo plenamente", visando mensurar a percepção dos dirigentes em relação aos elementos da gestão estratégica do conhecimento.

\section{RESULTADOS E DISCUSSÃO}


Cada questão teve por intuito medir um atributo específico, quinze questões medindo o SECI e outras dez questões medindo as dimensões estratégicas. A fim de validar o questionário, denotando até que ponto os constructos estavam medindo o que se esperava, foi realizado um pré-teste do questionário, com a finalidade de verificar a confiabilidade (alpha de Cronbach), o qual resultou num coeficiente alpha de 0,9068, um bom resultado perante a literatura (HAIR et al., 2005).

A primeira etapa para coleta de dados consistiu em uma pesquisa realizada com os administradores das incubadoras, visando obter as informações para estabelecer contato com todas as empresas instaladas no Ciatec e ParqTec.

Identificadas as empresas, foi enviada uma carta de apresentação da pesquisa aos dirigentes das empresas instaladas nas incubadoras, via e-mail; simultaneamente, foi feito contato com os dirigentes por telefone para frisar o interesse da pesquisa e sua colaboração.

Após essa etapa, procedeu-se então à coleta de dados por meio do envio de um questionário fechado, via e-mail, aos dirigentes das empresas. O questionário foi elaborado por meio da ferramenta formulários do Microsoft Word, a fim de agilizar o processo de preenchimento do questionário. O processo de coleta de todos os questionários enviados teve a duração de aproximadamente cinco meses.

Com base nas respostas em escala Likert (intervalar), foi realizada a análise de correspondência, o teste binomial e a análise de correlação, que permitiu avaliar a associação entre as variáveis investigadas (HAIR JR et al., 2005a).

Dessa forma, mediante a validação do questionário, foi possível analisar todas as variáveis (fatores SECI e dimensões estratégicas). São apresentados, assim, quais elementos estão relacionados ou não com os aspectos estratégicos no ambiente das incubadoras de empresas, permitindo a proposição de mecanismos que contribuam com a gestão estratégica do conhecimento nas MPE residentes em incubadoras.

Dentre as 39 empresas pesquisadas, foram invalidados os questionários em quatro empresas do Ciatec e cinco do ParqTec, pois estas eram compostas por apenas 1 pessoa, impossibilitando as inferências sobre gestão do conhecimento. Em outras cinco empresas do Ciatec não foi possível estabelecer contato; e, por fim, quatro empresas do Ciatec optaram por não participar da pesquisa. 
Totalizou-se assim uma amostra restante de 21 empresas, onze empresas instaladas no

ParqTec, dez empresas do Ciatec; a taxa de questionários aceitos sobre a amostra inicial de 16 empresas do ParqTec corresponde a 68,75\% e da amostra de 23 empresas do Ciatec é 43,48\%.

Perante as respostas coletadas, foi obtido um alpha equivalente a 0,8426 , para um questionário composto por 25 questões, conforme APÊNDICE, e uma amostra de 21 empresas instaladas nas incubadoras.

A comparação entre as incubadoras perante os elementos da gestão estratégica do conhecimento foi obtida por meio do teste binomial, que é usado para fazer inferências de diferenças entre duas populações, baseadas em dados de duas amostras aleatórias. Assim, os dados experimentais utilizados pelo teste são as frequências relativas $p$ e $q$ referentes às duas alternativas possíveis naquele determinado experimento. A freqüência esperada para $p$ e $q$, em caso de igualdade perfeita, seria $1 / 2$ para ambos. Como, num experimento, dificilmente $p$ é igual a $q$, o teste avalia, em última análise, até que ponto os valores de $p$ e $q$ podem diferir, sem deixarem de ser estatisticamente iguais; dessa forma, o teste fornece o $p$-value que informa se a proporção amostral de diferenças é significativa, isto é, se $\mathrm{H}_{0}$ for a hipótese verdadeira (CAMPOS, 2001).

Nesse contexto, o teste binomial foi utilizado como método de análise de proporção que compara as visões e as opiniões entre as incubadoras diferentes a respeito das mesmas questões.

\section{Hipótese Científica:}

$\mathrm{H}_{0}$ : Proporções de "discordância", "parcial" e "concordância" são iguais para os incubadoras de São Carlos e Campinas.

$\mathrm{H}_{1}$ : Proporções de "discordância", "parcial" e "concordância" são diferentes para os incubadoras de São Carlos e Campinas.

Hipótese Estatística:

$$
H_{0}:\left(\begin{array}{l}
P 1_{p 1} \\
P 2_{p 1} \\
P 3_{p 1}
\end{array}\right)=\left(\begin{array}{l}
P 1_{p 2} \\
P 2_{p 2} \\
P 3_{p 2}
\end{array}\right) \quad H_{1}:\left(\begin{array}{l}
P 1_{p 1} \\
P 2_{p 1} \\
P 3_{p 1}
\end{array}\right) \neq\left(\begin{array}{l}
P 1_{p 2} \\
P 2_{p 2} \\
P 3_{p 2}
\end{array}\right)
$$

Tal que P1 é a proporção de empresas incubadas que discordam, P2 é a proporção de empresas incubadas com opinião parcial e P3 é a proporção de empresas incubadas que concordam. 
A Tabela 1 mostra o comportamento de cada questão perante a comparação entre as duas incubadoras: ParqTec ( $p 1)$ e Ciatec ( $p 2)$.

\section{Tabela 1}

Comparação: São Carlos e Campinas

\begin{tabular}{ccccc}
\hline Questões & $\boldsymbol{p}$-value $\mathbf{P 1}$ & $\boldsymbol{p}$-value $\mathbf{P 2}$ & $\boldsymbol{p}$-value $\mathbf{P 3}$ & Resultado \\
\hline 1 & 0,943 & $*$ & 0,943 & Aceito \\
2 & 0,279 & 0,015 & 0,269 & Rejeito \\
3 & 0,476 & 0,593 & 0,890 & Aceito \\
4 & 0,696 & 0,696 & 0,505 & Aceito \\
5 & 0,329 & $*$ & 0,329 & Aceito \\
6 & 0,407 & 0,476 & 0,801 & Aceito \\
7 & $*$ & 0,476 & 0,476 & Aceito \\
8 & 0,217 & 0,050 & 0,835 & Rejeito \\
9 & 0,034 & 0,329 & 0,015 & Rejeito \\
10 & 0,593 & 0,476 & 0,890 & Aceito \\
11 & 0,020 & 0,314 & 0,284 & Rejeito \\
12 & 0,217 & 0,269 & 0,864 & Aceito \\
13 & 0,072 & 0,476 & 0,256 & Aceito \\
14 & 0,157 & 0,119 & 0,757 & Aceito \\
15 & 0,104 & 0,537 & 0,269 & Aceito \\
16 & 0,314 & 0,943 & 0,407 & Aceito \\
17 & 0,466 & 0,916 & 0,528 & Aceito \\
18 & 0,283 & 0,943 & 0,476 & Aceito \\
19 & 0,696 & 0,943 & 0,757 & Aceito \\
20 & 0,329 & 0,269 & 0,537 & Aceito \\
21 & 0,217 & $*$ & 0,217 & Aceito \\
22 & 0,217 & 0,525 & 0,528 & Aceito \\
23 & 0,593 & 0,916 & 0,757 & Aceito \\
24 & $*$ & 0,757 & 0,757 & Aceito \\
25 & $*$ & 0,943 & 0,943 & Aceito \\
\hline
\end{tabular}

p-value $\leq 0,05$

$\mathrm{O}$ asterisco $(*)$ representa que as respostas das empresas foram exatamente as mesmas

Fonte: Elaborado pelo autor.

Observa-se que apenas quatro questões rejeitaram $\mathrm{H}_{0}$, mostrando que as proporções de "discordância", "concordância" e "parcial" são diferentes para as incubadoras de São Carlos e Campinas no que concerne a essas quatro questões.

As questões rejeitadas foram 2, 8, 9 e 11, as quais correspondem às variáveis: treinamento, interações coletivas e virtuais, banco de dados e elaboração de relatórios, respectivamente. Pode-se observar que uma delas está inserida na fase de socialização e as demais na combinação.

As empresas residentes no Parque Científico Tecnológico de Campinas (Ciatec) demonstraram que a prática de treinamento, por meio de cursos extras, é pouco empregada, provavelmente pelo fato de serem empresas que tiveram sua fundação recente em relação 
àquelas do ParqTec, e por isso anseiam que seus funcionários já tenham uma qualificação alinhada à sua função, sem necessitar de investimentos extras da empresa no treinamento de funcionários. Ademais, o Ciatec é uma fundação recente, denotando uma estrutura que está em crescimento quanto à organização de treinamentos e cursos para as empresas residentes.

As interações coletivas e virtuais, por sua vez, são mais empregadas pelo Ciatec, o que pode ser explicado pelo maior número de funcionários das empresas, pois, no Ciatec, das dez empresas pesquisadas, $50 \%$ possuem mais de cinco funcionários; então, as interações coletivas e virtuais tornam-se indispensáveis para a execução dos projetos; dentre as onze empresas entrevistadas do ParqTec, por outro lado, apenas $20 \%$ possuem mais de cinco funcionários, acarretando interações muito mais individualizadas.

Embora mais de 50\% das empresas instaladas no ParqTec concordem com o acesso ao banco de dados da empresa, as empresas investigadas do Ciatec apresentaram 100\% de utilização dos bancos de dados, o que denotou na rejeição da hipótese $\mathrm{H}_{0}$; o tamanho das empresas segundo o critério por número de funcionário pode explanar esse fato, mas é provável que a cultura de incentivo ao acesso aos bancos de dados seja muito mais intensa nas empresas do Ciatec.

Por outro lado, a elaboração de relatórios é uma prática mais ativa no ParqTec, tornando assim o conhecimento sistematizado disponível para necessidades futuras, evitando a perda de tempo em tarefas que já foram executadas em projetos passados.

$\mathrm{O}$ teste binomial permitiu que se indicassem as principais divergências entre as empresas pesquisadas no ParqTec e do Ciatec, e observou-se que as diferenças são poucas entre os dois grupos investigados. Como foi verificado pela indicação do asterisco na Tabela 1 , houve seis casos dentre os 75 , correspondendo a $8 \%$ de respostas exatamente iguais entre as incubadoras, e apenas 5,33\% de questões que demonstraram diferenças entre os grupos. Ademais, as variáveis que compõem a fase combinação foram aquelas que geraram a maior divergência entre o ParqTec e o Ciatec.

\section{CONSIDERAÇÕES FINAIS}

Com base, nas diferenças entre as incubadoras no que concerne à gestão estratégica do conhecimento, é possível denotar alguns argumentos que explicam esse comportamento. Primeiramente, tem-se a heterogeneidade das empresas, que, além de estarem em ambientes 
PUC Minas

distintos e embora possuam recursos similares, apresentam, muitas vezes, diferenças consideráveis em estilo de gestão, recursos financeiros, tipo de produto ou serviço, tipo de processo de desenvolvimento, entre outros aspectos, característicos das empresas.

Além disso, a estratégia de muitas das empresas é a sobrevivência do negócio, e foi possível observar casos de empresas recém-formadas em que as despesas ainda eram maiores que as receitas, portanto a lucratividade ainda não era uma realidade, dificultando sobremaneira a inferência de práticas como o treinamento que exige um ambiente mais estruturado e consolidado; e, surpreendentemente, apesar dessas restrições, as inserções informais de mecanismos de gestão estratégica do conhecimento, como investimento em sistemas integrados de comunicação, ocorrem no contexto dessas empresas.

Nesse âmbito, observa-se que as relações estabelecidas entre o processo de gestão do conhecimento SECI e as dimensões estratégicas são distintas nas incubadoras, embora sejam ambientes (incubadoras) que teoricamente possuem algumas características similares, e as peculiaridades individuais prevalecem quando se analisa a gestão do conhecimento.

Alguns mecanismos poderiam ser empregados pelas empresas do ParqTec, com o intuito de configurar uma gestão estratégica do conhecimento mais efetiva:

- coletar e armazenar o maior número de informações e conhecimentos de projetos, produtos, serviços prestados anteriormente, a fim de que, na replicação de algum projeto, um tempo que já foi despendido para elaborá-lo em uma situação anterior não seja desperdiçado, tornando o acesso a essa base de dados uma rotina;

- incentivar a busca por informações nas bases de dados das empresas;

- incentivar interações intra e inter equipes virtuais, coletivizando-as.

Algumas sugestões são apresentadas para a melhoria do processo de gestão estratégica do conhecimento nas empresas instaladas no Ciatec:

- $\quad$ subsidiar treinamentos aos funcionários;

- coletar e armazenar o maior número de informações e conhecimentos de projetos, produtos, serviços prestados anteriormente e tornar a elaboração de relatórios uma prática rotineira;

- incentivar a utilização do conhecimento nas rotinas organizacionais, permitindo que seja agregado valor aos novos conhecimentos.

É possível denotar que algumas das dimensões estratégicas, como cultura, práticas de recursos humanos e sistemas de informação, são funções de apoio à criação, ao compartilhamento, ao armazenamento e à incorporação do conhecimento e devem ser 
exploradas ao máximo para configurar uma gestão mais efetiva. É importante destacar, contudo, algumas limitações encontradas nesta pesquisa e sugestões para trabalhos futuros, aspectos que são discutidos a seguir.

Embora tenha abrangido vinte e uma empresas instaladas em duas incubadoras, amostra significativa perante a população-alvo, este foi um fator limitador para uma análise quantitativa, impossibilitando a generalização dos resultados. A análise quantitativa, por sua vez, também se evidencia como um limitante, uma vez que as organizações e seus contextos não se apresentam como uma ciência exata.

Em trabalhos futuros sugerem-se estudos que contemplem a realidade das empresas incubadas, permitindo que práticas e ferramentas organizacionais, em sua maioria, derivadas das grandes empresas sirvam de base para o aprimoramento e a adequação de técnicas compatíveis com a realidade de pequenas empresas. Vale ressaltar que, neste estudo, priorizou-se a análise dos dois grupos de empresas instaladas nas incubadoras, e assim pôde ser realizado um estudo pontual e em profundidade em cada empresa, explorando os mecanismos qualitativos de gestão estratégica do conhecimento.

\section{Referências}

BENÍTEZ, Z. R.; RODRIGUEZ y RODRIGUEZ, M. V. A gestão estratégica do conhecimento e a relutância dos profissionais em compartilhar o conhecimento organizacional. Sustainable Business International Journal, n.1, v. 1, p.1-26, 2004.

BEIJERSE, R. P. Questions in knowledge management: defining and conceptualising a phenomenon. Journal of Knowledge Management, v. 3, n. 2, p. 94-109, 1999.

BRASIL. Lei Federal n. 10.973, de 2 de dezembro de 2004. Dispõe sobre incentivos à inovação e à pesquisa científica e tecnológica no ambiente produtivo e dá outras providências. Disponível em: <http://www.receita.gov.br/legislacao/leis/2004/lei10973.htm> Acesso em: 10 fev. 2005.

CAMPOS, G. M. Estatística prática para docentes e pós-graduandos. Disponível em: <http://www.forp.usp.br/restauradora/gmc/gmc_livro/gmc_livro_cap19.html>. Acesso em: 5 nov.2006. 2001.

CAMPOS, M. Z. C. R.; FERREIRA, M. A. T; SILVA, S. M. Transferência do conhecimento tecnológico gerado em projetos de P\&D no setor elétrico brasileiro: o caso Eletronorte. Revista Economia \& Gestão, v. 9, n. 21, p.100-119, 2009.

CARDOSO, L. M. G. P. P. A. Gerir conhecimento e gerar competitividade: estudo empírico sobre a gestão do conhecimento e seu impacto no desempenho organizacional. 2003. 587 f. Tese (Doutorado em Psicologia) - Universidade de Coimbra, Faculdade de Psicologia e Ciências da Educação, Coimbra. 
CERVO, A. L.; BERVIAN, P. A. Metodologia científica: para uso dos estudantes universitários. 3. ed. São Paulo: McGraw-Hill do Brasil, 1983.

COHENDET, P.; STEINMUELLER, W. E. The codification of knowledge: a conceptual and empirical exploration. Industrial and Corporate Change, v. 9, n. 2, p. 195-209, 2000.

COWAN, R.; DAVID, P. A.; FORAY, D. The explicit economics of knowledge codification and tacitness. Industrial and Corporate Change, v. 9, n. 2, p.211-253, 2000.

CRUZ, C. A. Gestão estratégica do conhecimento: estudo exploratório em empresas instaladas nos parques tecnológicos do Estado de São Paulo. 2007. 164 f. Dissertação (Mestrado em Engenharia de Produção) - Escola de Engenharia de São Carlos, Universidade de São Paulo, São Carlos.

DANE, F. C. Research methods. Belmont - CA: Brooks/Cole, 1990.

DAVENPORT, T. H.; DE LONG, D. W.; BEERS, M. C. Successful knowledge management projects. Sloan Management Review, v.39, n. 2, p.43-57, 1998.

FLEURY, M. T. L.; OLIVEIRA JR., M. M. Gestão Estratégica do Conhecimento. São Paulo: Atlas, 2001.

GIL, A. C. Como elaborar projetos de pesquisa. 4. ed. São Paulo: Atlas, 2002.

HAIR JR., J. F.; ANDERSON, R. E.; TATHAM, R. L.; BLACK, W. C. Análise multivariada de dados. Porto Alegre: Bookman, 2005a.

HAIR JR., J. F.; BABIN, B.; MONEY, A. H.; SAMOUEL, P. Fundamentos de métodos de pesquisa em administração. Porto Alegre: Bookman, 2005b.

HANSEN, M. T.; NOHRIA, N.; TIERNEY, T. What's your strategy for managing knowledge? Harvard Business Review, v. 77, n. 2, p.106-116, mar./abr. 1999.

HITT, M. A.; IRELAND, R. D.; HOSKINSSON, R. E. Administração Estratégica: competitividade e globalização. 4. ed. São Paulo: Pioneira Thomson Learning, 2002.

IBGE - Instituto Brasileiro de Geografia e Estatística. Disponível em <www.ibge.gov.br>. Acesso em: $1^{\circ}$ ago. 2005.

KROGH, G. Care in knowledge creation. California Management Review, v. 40, n. 3, p. 133-153, 1998.

LAKATOS, E. M.; MARCONI, M. A. Metodologia do trabalho científico. 4. ed. São Paulo: Atlas, 1992.

LEONARD-BARTON, D. Wellspring of Knowledge. Boston, MA: Harvard Business School Press, 1998.

LEONE, N. M. C. P. G. As especificidades das pequenas e médias empresas. Revista de Administração, São Paulo, v. 34, n. 2, p.91-94, 1999. 
LIMA, E.; ZOSCHKE, A. C. K. Relações dos Dirigentes e Gestão Estratégica de Pequenas e Médias Empresas. RAI - Revista de Administração e Inovação, v. 4, p. 150-164, 2007. Disponível em: <http://www.revista-rai.inf.br/ojs-2.1.1/index.php/rai/issue/view/20>. Acesso em: 25 fev. 2010.

MATIAS-PEREIRA, J.; KRUGLIANSKAS, I. Gestão da Inovação: a lei de inovação tecnológica como ferramenta de apoio às políticas industrial e tecnológica do Brasil. RAEeletrônica, v. 4, n. 2, art.18, jul./dez. 2005.

MEDEIROS, C. A. F. Comprometimento organizacional: um estudo de suas relações com as características organizacionais e desempenho nas empresas hoteleiras. 2003. $166 \mathrm{f}$. Tese (Doutorado em Administração) - Universidade de São Paulo, Faculdade de Economia, Administração e Contabilidade, São Paulo.

MEDEIROS, J. A.; MEDEIROS, L. A.; MARTINS, T.; PERILO, S. Polos, parques e incubadoras: a busca da modernização e competitividade. CNPq, SCT//PR, IBICT, SENAI. Brasília, 1992.

NONAKA, I. A dynamic theory of organizational knowledge creation. Organization Science, v. 5, n. 1, p. 14-37, 1994.

NONAKA, I.; KONNO, N. The concept of ba: building a foundation for knowledge creation . California Management Review, v.40, n.3, p.40-55. 1998.

NONAKA, I.; PELTOKORPI, V.; TOMAE, H. Strategic knowledge creation: the case of Hamamatsu Photonics. International Journal Techonology Management, v. 30, n. 3/4, p.248-264, 2005.

NONAKA, I.; TAKEUCHI, H. Criação de Conhecimento na Empresa: como as empresas japonesas geram a dinâmica da inovação. 13. ed., Rio de Janeiro: Elsevier, 1997.

NONAKA, I. The knowledge-creating Company. Harvard Business Review, v. 69, n. 6, p. 2-9, nov./dec. 1991.

NONAKA, I.; TOYAMA, R.; KONNO, N. SECI, Ba and Leadership: a unified model of dynamic knowledge creation. Long Range Planning, v. 33, p. 5-34, 2000.

NONAKA, I.; TOYAMA, R.; NAGATA, A. A Firm as a Knowledge-creating Entity: a new perspective on the Theory of the firm. Industrial and Corporate Change, v. 9, n. 1, p. 1-20, 2000 .

NONAKA, I.; UMEMOTO, K.; SENOO, D. From information processing to knowledge creation: a paradigm shift in business management. Technology in Society, v. 18, n. 2, p. 203-218, 1996.

ORSI, A. Incorporação de bases externas do conhecimento: a gestão do conhecimento nas fusões e aquisições de empresas. 2003. (número de folhas) Dissertação (Mestrado em Administração) - Universidade de São Paulo, Faculdade de Economia, Administração e Contabilidade, São Paulo.

PARKER, L. D.; RITSON, P. A. Fads, stereotypes and management gurus: fayol and follett today. Management Decision, Wagon Lane, v. 43, n. 10, p. 1335-1357, 2005. 
PORTER, M. E. Competitive Strategy. New York: Simon \& Schuster, 2003.

PRAHALAD, C. K.; HAMEL, G. The core competence of the corporation. Harvard Business Review, v. 68, n. 3, p.79-91, 1990.

PREVIDELLI, J. J.; MEURER, V. Gestão da micro, pequena e média empresa no Brasil: uma abordagem multidimensional. Maringá: UNICORPORE, 2005.

RODRIGUEZ y RODRIGUEZ, M. V. Gestão do Conhecimento: reinventando a empresa para uma sociedade baseada em valores intangíveis. Rio de Janeiro: IBPI Press, 2001.

SANTIAGO JR., J. R. S. O Desenvolvimento de uma Metodologia para a Gestão do Conhecimento em uma Empresa de Construção Civil. 2002. 127 f. Dissertação (Mestrado em Engenharia de Produção) - Universidade de São Paulo, Escola Politécnica, São Paulo.

SCHULZ, M. The uncertain relevance of newness: organizational learning and knowledge flows. Academy of Management Journal, v. 44, n. 4, p.661-681, 2001.

SILVA, S. L. Gestão do conhecimento: uma revisão crítica orientada pela abordagem da criação do conhecimento. Ciência da Informação, v. 33, n. 2, p.143-151, 2004.

SILVA, S. L. Proposição de um modelo para caracterização das conversões do conhecimento no processo de desenvolvimento de produtos. 2002. $231 \mathrm{f}$. Tese (Doutorado em Engenharia Mecânica) - Universidade de São Paulo, Escola de Engenharia de São Carlos, São Carlos.

STOREY, J.; QUINTAS, P. Knowledge Management and HRM. In: STOREY, J. (Org). Human Resource Managemen: A Critical Text. Great Britain: Thomson Learning, p.339363, 2001.

TEECE, D. J. Capturing value from knowledge assets: the new economy, markets for knowhow, an intangible assets. California Management Review, Reprint Series, v. 40, n. 3, p. 52 79, 1998.

TERENCE, A. C. F. Planejamento estratégico como ferramenta de competitividade na pequena empresa. 2002. 253 f. Dissertação (Mestrado em Engenharia de Produção) Universidade de São Paulo, Escola de Engenharia de São Carlos, São Carlos.

TERRA, J. C. C. Gestão do Conhecimento: o grande desafio empresarial: uma abordagem baseada no aprendizado e na criatividade. São Paulo: Negócio Editora, 2001.

TORKOMIAN, A. L. V. Estrutura de polos tecnológicos: um estudo de caso. 1992. $231 \mathrm{f}$. Dissertação (Mestrado em Administração) - Universidade de São Paulo, Faculdade de Economia e Administração, São Paulo.

VASCONCELOS, F. C. Da gestão do conhecimento à gestão da ignorância: uma visão coevolucionária. Revista de Administração de Empresas, v. 41, n. 4, p. 98-102, 2001.

ZACK, M. H. Developing a knowledge Strategy. California Management Review, v. 41, n. 3, p.125-145. 1999. 
WERNERFELT, B. A resource-based view of the firm. Strategic Management Journal, v. 5, p. 171-180, 1984.

WREN, D. A. Ideias de administração: o pensamento clássico. São Paulo: Ática, 2007.

\section{Apêndice - Questionário para os dirigentes}

INSTRUÇÕES: Para responder a questão, deve-se assinalar o número mais adequado dentro da escala, segundo a visão da organização, com relação à afirmação colocada, como o exemplo abaixo.

Ex. Seus clientes estão satisfeitos com o serviço prestado

Discordo Discordo de Discordo Concordo Concordo Concordo com Concordo
Totalmente praticamente ligeiramente parcialmente ligeiramente praticamente
tudo
\begin{tabular}{|c|c|c|c|c|c|c|}
\hline 1 & 2 & 3 & 5 & 6 & 7 \\
tudalmente
\end{tabular}

Nesse exemplo, nota-se que a satisfação dos clientes é parcial (4) em relação aos serviços prestados.

1. Quando um funcionário está em dúvida sobre determinada atividade de trabalho, uma de suas primeiras ações é recorrer a um colega de trabalho.

2. A empresa contrata cursos e treinamentos para seus funcionários, sempre que surge um novo projeto no qual o funcionário deverá desempenhar uma atividade diferente daquela até então exercida.

3. Após aprimorarem seus conhecimentos em cursos e treinamentos, os funcionários interagem sobre os conceitos aprendidos.

4. Os funcionários estão sempre a par do crescimento da empresa e de suas necessidades de qualificação, buscando se especializar mesmo quando não há qualquer subsídio da empresa.

5. Os projetos de trabalho são realizados, em sua maioria, por equipes que executam suas atividades e análises, através de interações conjuntas.

6. É comum entre os funcionários o uso de metáforas e analogias para explicar ideias e atividades.

7. Novas ideias são valorizadas. Há permissão para discussão de ideias que a princípio pareçam irrelevantes.

8. Na empresa há grupos de discussão os quais se relacionam por meio de ambientes virtuais, tais como: fóruns virtuais e grupos de e-mails.

9. $\mathrm{O}(\mathrm{s})$ banco (s) de dados da empresa é (são) intensamente acessado(s), tanto para armazenar como fornecer dados/informações a todos na empresa.

10. A empresa provê sistemas integrados que incentivam diálogos por meio de intranet e internet, tais como e-mails, MSN, CRM.

11. A empresa exige que, após a conclusão de projetos e atividades, os funcionários redijam relatórios sobre estes.

12. Os ambientes virtuais de comunicação (intranet, internet) são utilizados mais como veículos de comunicação entre dois indivíduos em vez de ser entre grupos. 
PUC Minas

13. Todos os funcionários estão aptos a dizerem, se questionados, qual a meta e a missão da empresa.

14. Os funcionários, em sua maioria, apresentam mais know-how técnico, isto é, são mais especialistas, do que generalistas.

15. Quando um funcionário começa a realizar uma nova atividade que ele nunca exerceu, ele tenta desempenhá-la, primeiramente, por meio da prática, isto é, aprender-fazendo.

16. Os funcionários têm consciência de que, se contribuírem com o alcance da meta da empresa, por exemplo, criando um produto/serviço inovador, eles serão reconhecidos (valorizados).

17. A experimentação é estimulada. Há liberdade para tentar e falhar.

18. As pessoas estão preocupadas com toda a organização e não apenas com sua área de trabalho, buscando soluções conjuntas.

19. Os cursos, especializações e títulos obtidos pelos funcionários são valorizados.

20. Os recém-contratados, antes de iniciarem suas atividades, são treinados pelos funcionários mais antigos à empresa.

21. Existem esquemas de participação nos lucros, premiação e recompensas por resultados e contribuições.

22. Na contratação busca-se a diversidade (personalidades, experiências, cultura, etc.), em detrimento do know-how técnico.

23. Todos os sistemas de informação estão integrados, assim é possível que qualquer área da empresa tenha acesso ao conteúdo das demais via intranet.

24. Um dos maiores investimentos realizados pela empresa, anualmente, dedica-se aos sistemas de informação.

25. A comunicação, por meio das redes de comunicação, é eficiente em todos os sentidos (de cima para baixo, de baixo para cima e entre as diferentes áreas). 